\title{
General Monogamy Inequality for Bipartite Qubit Entanglement
}

\author{
Tobias J. Osborne $1, *$ and Frank Verstraete $2, \oplus$ \\ ${ }^{1}$ Department of Mathematics, University of Bristol, University Walk, Bristol BS8 1TW, United Kingdom \\ ${ }^{2}$ Institute for Quantum Information, California Institute of Technology, Pasadena, CA 91125, USA
}

(Dated: October 22, 2018)

\begin{abstract}
We consider multipartite states of qubits and prove that their bipartite quantum entanglement, as quantified by the concurrence, satisfies a monogamy inequality conjectured by Coffman, Kundu, and Wootters. We relate this monogamy inequality to the concept of frustration of correlations in quantum spin systems.
\end{abstract}

PACS numbers: 03.65.Bz, 89.70.+c

Quantum mechanics, unlike classical mechanics, allows the existence of pure states of composite systems for which it is not possible to assign a definite state to two or more subsystems. States with this property are known as entangled states. Entangled states have a number of remarkable features, a fact which has inspired an enormous literature in the years since their discovery. These properties have led to suggestions that the propensity of multipartite quantum systems to enter nonlocal superposition-states might be the defining characteristic of quantum mechanics [1, 2].

It is becoming clear that entanglement is a physical resource. The exploration of this idea is a central goal in the burgeoning field of quantum information theory. As a consequence, the study of the mathematics underlying entanglement has been a very active area and has led to many operational and information-theoretic insights. As for now, only the pure-state case of entanglement shared between two parties is thoroughly understood and quantified; progress on the multipartite setting has been much slower.

A key property, which maybe as fundamental as the nocloning theorem, has been discovered recently in the context of multipartite entanglement: entanglement is monogamous [3, 4]. More precisely, there is an inevitable tradeoff between the amount of quantum entanglement that two qubits $A$ and $B$, in Alice's and Bob's possession, respectively, can share and the quantum correlation that Alice's same qubit $A$ can share with Charlie, a third party, $C$ [3]. In the context of quantum cryptography, such a monogamy property is of fundamental importance because it quantifies how much information an eavesdropper could potentially obtain about the secret key to be extracted. The constraints on shareability of entanglement lie also at the heart of the success of many information-theoretic protocols, such as entanglement distillation.

In the context of condensed matter physics, the monogamy property gives rise to the frustration effects observed in, e.g., Heisenberg antiferromagnets. Indeed, the perfect ground state for an antiferromagnet would consist of singlets between all interacting spins. But, as a particle can only share one unit of entanglement with all its neighbours (this immediately follows from the dimension of its local Hilbert space), it will try to spread its entanglement in an optimal way with all its neighbours leading to a strongly correlated ground state. The tools developed in this Letter will allow us to turn such qualitative statements into quantitative ones.

The problem of fully quantifying the constraints on distributed entanglement should be seen as analogous to the $N$-representability problem for fermions [5]. This is because, just as is the case for fermions, if the constraints on distributed entanglement were known explicitly then this would render trivial [6] the task of computing the groundstate energy of condensed-matter systems. The results of this Letter represent the first step towards the full quantification of the constraints on distributed entanglement.

The main result of this Letter is a proof of the longstanding conjecture of Coffman, Kundu, and Wootters [3] that the distribution of bipartite quantum entanglement, as measured by the tangle $\tau$, amongst $n$ qubits satisfies a tight inequality:

$\tau\left(\rho_{A_{1} A_{2}}\right)+\tau\left(\rho_{A_{1} A_{3}}\right)+\cdots+\tau\left(\rho_{A_{1} A_{n}}\right) \leq \tau\left(\rho_{A_{1}\left(A_{2} A_{3} \cdots A_{n}\right)}\right)$,

where $\tau\left(\rho_{A_{1}\left(A_{2} A_{3} \cdots A_{n}\right)}\right)$ denotes the bipartite quantum entanglement measured by the tangle across the bipartition $A_{1}: A_{2} A_{3} \cdots A_{n}$. This inequality (which we shall henceforth refer to as the $C K W$ inequality) has been established in the case of three qubits. However, the case of $n$ qubits was still open [24]. In this Letter we establish Eq. (1) for arbitrary numbers $n$ of qubits.

The outline of this Letter is as follows. We begin by introducing and defining the quantum correlation measures we study throughout this Letter. Following this we reduce the CKW inequality to a statement pertaining to quantum correlation measures for a pure tripartite system consisting of two qubits and a four-level quantum system. Such a system is, up to local unitaries, completely determined by its two-qubit reduced density operator. The proof will then be completed by showing that the one-way correlation measure [7, 8] of a mixed state of two qubits is always larger or equal than its tangle.

In our proof we have utilised a number of techniques. We derive a computable formula for the linear Holevo $\chi$ quantity for all qubit maps and also for the one-way correlation measure [8] for all two-qubit states.

To quantify mixed-state bipartite quantum correlations 
we study two measures. We also study one channel capacity measure. The first measure we consider is the tangle $\tau\left(\rho_{A B}\right)$ which is the square of the concurrence [3, 9, 10, 11, 12], $\tau\left(\rho_{A B}\right)=C^{2}\left(\rho_{A B}\right)$. The tangle measure pertains to bipartite quantum states $\rho_{A B}$ of a qubit $A$ and a $D$-level quantum sytem $B$. To define the tangle we introduce the following entropic measure, the linear entropy $S_{2}$, for single-qubit states $\rho$ [23]:

$$
\begin{aligned}
S_{2}(\rho) & \triangleq 2\left(1-\operatorname{tr}\left(\rho^{2}\right)\right) \\
& =4 \operatorname{det}(\rho) .
\end{aligned}
$$

The linear entropy $S_{2}$ is concave and unitarily invariant.

The tangle $\tau$ is now defined for any state $\rho_{A B}$ of the $2 \times D$ system via the roof construction (for operational motivations and further discussion of this construction see [9] and [13])

$$
\tau\left(\rho_{A B}\right) \triangleq \inf _{\left\{p_{x}, \psi_{x}\right\}} \sum_{x} p_{x} S_{2}\left(\operatorname{tr}_{B}\left(\psi_{x}\right)\right),
$$

where the infimum runs over all pure-state decompositions $\left\{p_{x}, \psi_{x}\right\}$ of $\rho_{A B}, \rho_{A B}=\sum_{x} p_{x} \psi_{x}$.

The second correlation measure we need is closely related to a one-way correlation measure [7, 8]. For any mixed state $\rho_{A B}$ of a $2 \times D$ bipartite quantum system we define

$$
I_{2}^{\leftarrow}\left(\rho_{A B}\right) \triangleq \max _{\left\{M_{x}\right\}}\left(S_{2}\left(\rho_{A}\right)-\sum_{x} p_{x} S_{2}\left(\rho_{x}\right)\right),
$$

where the maximum runs over all POVMs $\left\{M_{x}\right\}$ on Bob's system, $p_{x}=\operatorname{tr}\left(I_{B} \otimes M_{x} \rho_{A B}\right)$ is the probability of outcome $x$, and $\rho_{x}=\operatorname{tr}_{B}\left(I_{B} \otimes M_{x} \rho_{A B}\right) / p_{x}$ is the posterior state in Alice's subsystem.

The third measure we will need, the linear Holevo $\chi$ capacity, is a capacity measure for qubit channels $\Lambda$. This measure is related to the one-shot Holevo $\chi$ quantity and is defined by

$$
\chi_{2}(\rho ; \Lambda)=\max _{\left\{p_{x}, \psi_{x}\right\}}\left(S_{2}(\Lambda(\rho))-\sum_{x} p_{x} S_{2}\left(\Lambda\left(\psi_{x}\right)\right)\right),
$$

where $\rho$ is a qubit ensemble, $\Lambda$ is an arbitrary qubit channel (a trace-preserving completely-positive map), and the maximum runs over all pure state decompositions $\left\{p_{x}, \psi_{x}\right\}$ of $\rho, \rho=\sum_{x} p_{x} \psi_{x}$.

We now turn to the CKW inequality. Our strategy for proving Eq. (1) will be to prove it for states $\rho_{A B C}$ of two qubits $A B$, and a $2^{n-2}$-dimensional qudit $C$. The next step we use is to proceed via induction by successively partitioning the last qudit $C$ into two subsystems, a qubit $C_{1}$, and a $2^{n-3}$-dimensional qudit $C_{2}$, and establishing Eq. (1) for the (typically mixed) state $\rho_{A C_{1} C_{2}}$. Thus, the formula we will try to prove is the following

$$
\tau\left(\rho_{A(B C)}\right) \geq \tau\left(\rho_{A B}\right)+\tau\left(\rho_{A C}\right),
$$

for arbitrary states $\rho$ of a $2 \times 2 \times 2^{n-2}$ system $A B C$.
We begin by trying to prove Eq. (5) for pure states. In this case we can use the local-unitary invariance of $\tau\left(\rho_{A C}\right)$ to rotate the basis of subsystem $C$ into the local Schmidt basis $\left|u_{j}\right\rangle, j=1, \ldots, 4$, given by the eigenvectors of $\rho_{C}$. In this way we can regard the $2^{n-2}$-dimensional qudit $C$ as an effective 4-dimensional qudit. Therefore, it is sufficient to establish Eq. (5) for a $2 \times 2 \times 4$ system $A B C$.

Supposing we have proved the inequality Eq. (5) for pure states we can extend Eq. (5) to mixed states $\rho$. Consider the minimising decomposition $\left\{p_{x},\left|\psi_{x}\right\rangle\right\}$ for $\tau\left(\rho_{A(B C)}\right)$, and apply the inequality Eq. (5) to each term,

$$
\begin{aligned}
\tau\left(\rho_{A(B C)}\right) & =\sum_{x} p_{x} \tau\left(\rho_{A(B C)}^{x}\right), \\
& \geq \sum_{x} p_{x}\left(\tau\left(\rho_{A B}^{x}\right)+\tau\left(\rho_{A C}^{x}\right)\right), \\
& \geq \tau\left(\rho_{A B}\right)+\tau\left(\rho_{A C}\right),
\end{aligned}
$$

where $\rho_{A(B C)}^{x}=\left|\psi_{x}\right\rangle\left\langle\psi_{x}\right|$, and we have used the convexity of $\tau$ to arrive at the third line.

Now all that is required to establish the inequality Eq. (5) for an arbitrary system of $n$ qubits is to successively apply Eq. (5) to partitions of $C$ according to the inductive recipe outlined above. We illustrate this procedure for pure states $\rho$ of four qubits $A B C_{1} C_{2}$. Let $C=C_{1} C_{2}$ be a combined pair of qubits and apply Eq. (5),

$$
\begin{aligned}
\tau\left(\rho_{A(B C)}\right) & \geq \tau\left(\rho_{A B}\right)+\tau\left(\rho_{A C}\right), \\
& \geq \tau\left(\rho_{A B}\right)+\tau\left(\rho_{A C_{1}}\right)+\tau\left(\rho_{A C_{2}}\right),
\end{aligned}
$$

where we have applied the mixed-state version of the inequality Eq. (5) in the second line. It is straightforward to generalise this procedure to $n$ qubits.

We have now reduced the CKW inequality to an inequality for the tangle for pure states of a tripartite system $A B C$ of two qubits $A$ and $B$ and a four-level system $C$. In the case of pure states, $\rho_{A B}$ and $\rho_{A C}$ contain the same information (up to local unitaries); all possible POVM measurents at Bob's side induce all possible pure state decompositions of $\rho_{A C}$, and therefore the following monogamy relation holds (see also Koashi and Winter [7]):

$$
S_{2}\left(\rho_{A}\right)=\tau\left(\rho_{A(B C)}\right)=I_{2}^{\leftarrow}\left(\rho_{A B}\right)+\tau\left(\rho_{A C}\right) .
$$

By comparing Eq. (5) and Eq. (8) we see that in order to establish Eq. (5) it is sufficient to establish the inequality

$$
\tau\left(\rho_{A B}\right) \leq I_{2}^{\leftarrow}\left(\rho_{A B}\right)
$$

for all two-qubit states $\rho_{A B}$. As a first step toward proving this inequality, we will now derive a computable formula for $I_{2}^{\leftarrow}\left(\rho_{A B}\right)$.

Any bipartite quantum state $\rho_{A B}$ may be written as

$$
\rho_{A B}=\Lambda_{\rho} \otimes I_{B}\left(\left|r_{B^{\prime} B}\right\rangle\left\langle r_{B^{\prime} B}\right|\right),
$$

where $\left|r_{B^{\prime} B}\right\rangle$ is the symmetric two-qubit purification of the reduced density operator $\rho_{B}$ on an auxiliary qubit system $B^{\prime}$ and $\Lambda_{\rho}$ is a qubit channel from $B^{\prime}$ to $A$. It can now readily be seen that the one-way correlation measure 
$I_{2}^{\leftarrow}\left(\rho_{A B}\right)$ is equal to the one-shot channel capacity measure $\chi_{2}\left(\rho_{B} ; \Lambda_{\rho}\right)$ : all possible POVM measurements induce all convex decompositions of $\rho_{B^{\prime}}$.

The action of a qubit channel $\Lambda$ on a single qubit state $\rho=\frac{I+\mathbf{r} \cdot \boldsymbol{\sigma}}{2}$, where $\boldsymbol{\sigma}$ is the vector of Pauli operators, may be written as

$$
\Lambda(\rho)=\frac{I+(\mathbf{L r}+\mathbf{l}) \cdot \boldsymbol{\sigma}}{2},
$$

where $\mathbf{L}$ is a $3 \times 3$ real matrix and $\mathbf{l}$ is a three dimensional vector. In this Pauli basis, the possible decompositions of $\rho_{B}$ into pure states are represented by all possible sets of probabilities $\left\{p_{j}\right\}$ and unit vectors $\left\{\mathbf{r}_{j}\right\}$ for which $\sum_{j} p_{j} \mathbf{r}_{j}=\mathbf{r}_{B}$ where $\frac{I+\mathbf{r}_{B} \cdot \boldsymbol{\sigma}}{2}=\rho_{B}$. The linear entropy $S_{2}$, written in terms of the Bloch vector $\mathbf{r}$ of a two-qubit state, is given by $S_{2}\left(\frac{I+\mathbf{r} \cdot \boldsymbol{\sigma}}{2}\right)=1-|\mathbf{r}|^{2}$. In this way we see that

$Q(\mathbf{r})=S_{2}\left(\Lambda\left(\frac{I+\mathbf{r} \cdot \boldsymbol{\sigma}}{2}\right)\right)=1-(\mathbf{L r}+\mathbf{l})^{T}(\mathbf{L r}+\mathbf{l})$,

which is a quadratic form in the Bloch vector $\mathbf{r}$.

Substituting $\mathbf{r}_{j}=\mathbf{r}_{B}+\mathbf{x}_{j}$, one can easily check that the calculation of $\chi_{2}\left(\rho_{B} ; \Lambda_{\rho}\right)$ reduces to determining $\left\{p_{j}, \mathbf{x}_{j}\right\}$ subject to the conditions $\sum_{j} p_{j} \mathbf{x}_{j}=\mathbf{0}$ and $\left\|\mathbf{r}_{B}+\mathbf{x}_{j}\right\|=1$ maximizing

$$
\max _{\left\{p_{j}, \mathbf{x}_{j}\right\}} \sum_{j} p_{j} \mathbf{x}_{j}^{T} \mathbf{L}^{T} \mathbf{L} \mathbf{x}_{j}
$$

Let us, without loss of generality, assume that $\mathbf{L}^{T} \mathbf{L}$ is diagonal with diagonal elements $\lambda^{x} \geq \lambda^{y} \geq \lambda^{z}$. The constraints $\left\|\mathbf{r}_{B}+\mathbf{x}_{j}\right\|=1$ lead to the identities

$$
\left(\mathbf{x}_{j}^{x}\right)^{2}=1-\left\|\mathbf{r}_{B}\right\|^{2}-2 \mathbf{r}_{B}^{T} \mathbf{x}_{j}-\left(\mathbf{x}_{j}^{y}\right)^{2}-\left(\mathbf{x}_{j}^{z}\right)^{2} .
$$

Substituting this into (13), we get

$$
\begin{aligned}
& \chi_{2}\left(\rho_{B} ; \Lambda_{\rho}\right)=\lambda_{x}\left(1-\left\|\mathbf{r}_{B}\right\|^{2}\right)+ \\
& \quad \max _{\left\{p_{j}, \mathbf{x}_{j}\right\}} \sum_{j} p_{j}\left(\left(\lambda_{y}-\lambda_{x}\right)\left(\mathbf{x}_{j}^{y}\right)^{2}+\left(\lambda_{z}-\lambda_{x}\right)\left(\mathbf{x}_{j}^{z}\right)^{2}\right) .
\end{aligned}
$$

This expression is obviously maximised by choosing $\mathbf{x}_{j}^{z}=$ $\mathbf{x}_{j}^{y}=0$ for all $j$; the $\mathbf{x}_{j}^{x}$ then have to correspond to the roots of the equation $\left\|\mathbf{r}_{B}+\mathbf{x}_{j}\right\|=1$. There are exactly two such roots, showing that the the maximum $\lambda_{x}\left(1-\left\|\mathbf{r}_{B}\right\|^{2}\right)$ can be reached by an ensemble of two states.

As $S_{2}\left(\rho_{B}\right)=1-\left\|\mathbf{r}_{B}\right\|^{2}$, we therefore obtain the following computable expression for the linear Holevo $\chi$ capacity for qubit channels:

$$
\chi_{2}\left(\rho_{B} ; \Lambda\right)=\lambda_{\max }\left(\mathbf{L}^{T} \mathbf{L}\right) S_{2}\left(\rho_{B}\right) .
$$

From this expression we also obtain an expression for $I_{2}^{\leftarrow}\left(\rho_{A B}\right)$ via the correspondence Eq. (10).

Now that we have a formula for $I_{2}^{\leftarrow}\left(\rho_{A B}\right)$, we want to prove that it is always larger than or equal to $\tau\left(\rho_{A B}\right)$. First of all, we note that a local filtering operation of the form $\rho_{A B}^{\prime}=\frac{(\mathbb{I} \otimes B) \rho_{A B}(\mathbb{I} \otimes B)^{\dagger}}{\operatorname{tr}\left(\left(\mathbb{I} \otimes B^{\dagger} B\right) \rho_{A B}\right)}$ leaves $\mathbf{L}$ invariant and transforms

$$
S_{2}\left(\rho_{B}^{\prime}\right)=\frac{\operatorname{det}(B)^{2}}{\operatorname{tr}\left(\left(\mathbb{I} \otimes B^{\dagger} B\right) \rho_{A B}\right)^{2}} S_{2}\left(\rho_{B}\right) .
$$

It happens that $I_{2}^{\leftarrow}$ transforms in exactly the same way as the tangle does [14] (recalling that the tangle is the square of the concurrence). As there always exists a filtering operation for which $\rho_{B}^{\prime} \propto I_{2}$, we can assume, without loss of generality, that $S_{2}\left(\rho_{B}\right)=1$.

So let's consider $\rho_{A B}$ with $\operatorname{Tr}_{A}\left(\rho_{A B}\right)=\frac{1}{2} I$. As $\lambda_{\max }\left(\mathbf{L}^{T} \mathbf{L}\right)=\sigma_{\max }^{2}(\mathbf{L})$ where $\sigma_{\max }(\mathbf{L})$ is the largest singular value of $\mathbf{L}$, we want to prove that $\sigma_{\max }(\mathbf{L}) \geq$ $C\left(\rho_{A B}\right)$ where $C\left(\rho_{A B}\right)$ denotes the concurrence of $\rho_{A B}$. It has been proven in [15] that any mixed state of two qubits with associated $3 \times 3$ matrix $\mathbf{L}_{j k}=\operatorname{Tr}\left(\rho \sigma_{j} \otimes \sigma_{k}\right)$ can be written as a convex decomposition of rank-2 density operators all having the same $\mathbf{L}_{j k}$. As the concurrence is convex, the maximum concurrence for a given $\mathbf{L}$ will certainly be achieved for a rank- 2 density operator $\rho_{2}$. Next notice that any rank- 2 matrix $\rho_{2}$ can, up to local unitaries, be written as

$$
\rho_{2}=p|00\rangle\langle 00|+(1-p)| \psi\rangle\langle\psi| .
$$

Given the concurrence of $C(|\psi\rangle\langle\psi|)=C$, then obviously $C\left(\rho_{2}\right) \leq(1-p) C$. Let us now consider $\sigma_{\max }(\mathbf{L})$; this is the largest singular value of the sum of two matrices, one having singular values $[p, 0,0]$ and the other one having $(1-p)[C, C, 1]$ (corresponding to $|00\rangle$ and $|\psi\rangle$ ). Up to left and right multiplication by unitaries, $\mathbf{L}$ is then given by

$$
\mathbf{L}=(1-p)\left(\begin{array}{ccc}
C & 0 & 0 \\
0 & C & 0 \\
0 & 0 & 1
\end{array}\right)+p\left(\begin{array}{c}
\cos (\phi) \\
0 \\
\sin (\phi)
\end{array}\right) \mathbf{u}^{T}
$$

where $\mathbf{u}$ is a unit vector. Obviously, the $(2,2)$ element of this matrix is $(1-p) C$, which is certainly a lower bound for $\sigma_{\max }(\mathbf{L})$. This therefore implies that $I_{2}^{\leftarrow}\left(\rho_{A B}\right) \geq \tau\left(\rho_{A B}\right)$ for all two-qubit states $\rho_{A B}$, hence proving the CKW inequality Eq. (11.

The CKW inequality is likely to be useful in a number of contexts, allowing simplified proofs of no-broadcasting bounds and constraints for qubit multitap channel capacities. Perhaps the most interesting open problem at this stage is to generalise Eq. (1) to systems other than qubits and to the case where $A_{1}$ consists of more than one qubit. In both these cases the available generalisations of the tangle measure for quantum entanglement provably cannot yield entanglement sharing inequalities. It is an interesting open problem to work out an easily computable measure of quantum entanglement which will yield concrete useful bounds on the distribution of private correlations.

The CKW inequality may be immediately applied to study the entanglement for a wide class of complex quantum systems. Let us, for example, consider a translationinvariant state of a quantum spin $1 / 2$ system on a lattice 
with coordination number $d$. The CKW inequality implies that the concurrence $C(\rho)$ of the reduced density operator $\rho$ of two nearest neighbours satisfies $C(\rho) \leq$ $\left(1-\left\langle S_{\mathbf{n}}\right\rangle^{2}\right) / \sqrt{d}$, where $\left\langle S_{\mathbf{n}}\right\rangle$ is the magnetisation in the direction $\mathbf{n}$. Hence the CKW inequality provides a quantitative tool of assessing how far the mean-field energy will be from the exact one. Let's e.g. consider the Heisenberg Hamiltonian. As the overlap of a state $\rho$ with a singlet is bounded above by $(1+C(\rho)) / 2[15]$ and as the mean field energy per bond is given by $1 / 2$, the gap between mean field theory and and the exact ground state density [16] is bounded above by $\left(1-\left\langle S_{\mathbf{n}}\right\rangle^{2}\right) /(2 \sqrt{d})$. The classical result [17] that mean field theory becomes exact, i.e. $\rho$ is separable, when $d \rightarrow \infty$ is a limiting case of this inequality.

In a similar context, several investigations of the constraints on distributed entanglement have been carried out recently. We mention, for example, [18, 19]. The validity of some results of these papers were conditioned on the truth of the CKW inequality. As a consequence of this Letter it is now possible to regard these results as true.

In this Letter we have proved that the distribution of bipartite quantum entanglement is subject to certain shareability laws. It is tempting to think that such shareability constraints might hold for other quantum correlation quantities, such as the Bell violation of a bipartite Bell inequality. This is in fact the case; it has recently been discovered [20] that bipartite Bell violations cannot be distributed arbitrarily.

Finally, it is worth highlighting some classes of quantum states which saturate the CKW inequality. The classic example of a quantum state saturating the $\mathrm{CKW}$ inequality is the $W$-state

$$
|W\rangle=\frac{1}{\sqrt{n}}(|0 \cdots 01\rangle+|0 \cdots 10\rangle+\cdots+|1 \cdots 00\rangle) .
$$

The $W$-state has the property that the entanglement of any two spins is equal, but the entanglement of the spin $A_{1}$ is not maximal. One might ask if there are any states which saturate the CKW inequality which have the property that the spin $A_{1}$ at the focus is maximally entangled with the rest. In this way we could regard such a state as sharing out a full unit of entanglement with its neighbours. Such a state does indeed exist and is given by

$$
|\psi\rangle=\frac{1}{\sqrt{2}}|0\rangle|0 \cdots 0\rangle+\frac{1}{\sqrt{2}}|1\rangle|W\rangle
$$

In conclusion, we proved the Coffman-Kundu-Wootters monogamy inequality which quantifies the frustration of entanglement between different parties. The unique feature of this inequality is that it is valid for any multipartite state of qubits, irrespective of the underlying symmetries, which makes it much more general than de Finetti type bounds [21]. We also discussed the relevance of the monogamous nature of entanglement in quantum cryptography and in frustrated quantum spin systems.
We would particularly like to thank Michael Nielsen for introducing TJO to this problem and for providing a tremendous amount of encouragement and suggestions. Also, we are deeply indebted to Andreas Winter for extensive helpful suggestions and comments. We would also like to thank Bill Wootters for many encouraging discussions. Finally, TJO is grateful to the EU for support for this research under the IST project RESQ and also to the UK EPSRC through the grant QIPIRC. FV is grateful to the Gordon and Betty Moore Foundation.

*T.J.Osborne@bristol.ac.uk

$\dagger$ fverstraete@ @ist.caltech.edu

[1] E. Schrödinger, Proc. Camb. Philos. Soc. 31, 555 (1935).

[2] J. S. Bell, Physics 1, 195 (1964).

[3] V. Coffman, J. Kundu, and W. K. Wootters, Phys. Rev. A 61, 052306 (2000), quant-ph/9907047.

[4] B. M. Terhal (2003), quant-ph/0307120.

[5] A. J. Coleman and V. I. Yukalov, Reduced density matrices, vol. 72 of Lecture Notes in Chemistry (Springer-Verlag, Berlin, 2000).

[6] F. Verstraete and J. I. Cirac (2005), quant-ph/0505140.

[7] M. Koashi and A. Winter, Phys. Rev. A 69, 022309, 6 (2004), quant-ph/0310037.

[8] L. Henderson and V. Vedral, J. Phys. A 34, 6899 (2001), quant-ph/0105028.

[9] C. H. Bennett, D. P. DiVincenzo, J. A. Smolin, and W. K. Wootters, Phys. Rev. A 54, 3824 (1996), quant-ph/9604024.

[10] W. K. Wootters, Phys. Rev. Lett. 80, 2245 (1998), quant$\mathrm{ph} / 9709029$.

[11] S. Hill and W. K. Wootters, Phys. Rev. Lett. 78, 5022 (1997), quant-ph/9703041.

[12] T. J. Osborne (2002), quant-ph/0203087.

[13] A. Uhlmann, Open Syst. Inf. Dyn. 5, 209 (1998), quant$\mathrm{ph} / 9704017$.

[14] F. Verstraete, J. Dehaene, and B. De Moor, Phys. Rev. A 64, 010101(R) (2001), quant-ph/0011111.

[15] F. Verstraete and H. Verschelde, Phys. Rev. A 2, 022307 (2002), quant-ph/0203073.

[16] M. R. Dowling, A. C. Doherty, and S. D. Bartlett, Phys. Rev. A 70, 062113 (2004), quant-ph/0408086.

[17] R. F. Werner, Lett. Math. Phys. 17, 359 (1989).

[18] T. Roscilde, P. Verrucchi, A. Fubini, S. Haas, and V. Tognetti, Phys. Rev. Lett. 93, 167203 (2004), condmat/0404403.

[19] T. Roscilde, P. Verrucchi, A. Fubini, S. Haas, and V. Tognetti, Phys. Rev. Lett. 94, 147208 (2005), condmat/0412098.

[20] B. F. Toner (2006), quant-ph/0601172.

[21] R. König and R. Renner, J. Math. Phys. 46, 122108 (2005), quant-ph/0410229.

[22] C.-s. Yu and H.-s. Song, Phys. Rev. A 71, 042331 (2005), quant-ph/0501079.

[23] The Renyi 2-entropy $R$ is often denoted as $S_{2}$ in the literature. While the linear entropy is equivalent to the Renyi 2-entropy, $R=-\log \left(\operatorname{tr}\left(\rho^{2}\right)\right)$, the reader should note that they are not the same quantity. 
[24] A CKW-like inequality has been established for $n$ qubits for an entanglement-like quantity which is a lower bound for tangle [22]. That result does not imply the CKW inequality. 